\title{
The relationship of local to overall behavioral contrast
}

\author{
TIMOTHY L. CLEARY \\ Illinois College, Jacksonville, Illinois
}

(Frances K. McSweeney, Sponsor)

\begin{abstract}
Pigeons were presented with a three-component multiple schedule, with components appearing in an unpredictable order. After a baseline period in which all components were associated with reinforcement, a discrimination period followed in which one of the components was associated with extinction. Of the remaining two positive components, one was at times directly preceded by the extinction component, and one was not. Both overall positive contrast and local positive contrast were measured relative to a baseline produced each session, and both types of contrast were found. The association between overall and local contrast decreased as discrimination training progressed. The results agree with a precursor theory of the origin of overall contrast.
\end{abstract}

Positive behavioral contrast is an increase in the rate of responding in the presence of an $S+$ whenever an interacting $\mathbf{S}-$ is introduced. Two forms have been distinguished: overall and local contrast. Overall refers to an average rate increase in $S+$, whereas local refers to an increase in $\mathrm{S}+$ due to an immediately preceding $\mathrm{S}-$.

Behavioral contrast is a phenomenon of stimulus control that has been of some interest to researchers (see review by Williams, 1983). Part of the interest stems from the amplification of discrimination performance that the effect produces. For example, Terrace (1966) demonstrated that during a simple multiple-schedule discrimination between two colors, one associated with a variableinterval (VI) 60-sec schedule $(\mathrm{S}+$ ) and the other associated with an extinction (EXT) schedule $(\mathrm{S}-)$, the response rates during the presence of $S+$ were higher when following a presentation of the EXT component than when following the presentation of the S+ itself. A conceptually similar finding was reported by Pavlov (1927, Lecture XI) when a CS - preceded a CS + .

In Terrace's (1966) study, the average response rate in $\mathbf{S}+$ was compared with an average baseline rate, which was taken before an extinction schedule was introduced and overall positive contrast was found. The local positive contrast effect of the preceding extinction stimulus tended to disappear after prolonged training, even though an overall response rate elevation above baseline remained. Terrace's findings have generally been confirmed (Blough, 1988; Mackintosh, Little, \& Lord, 1972; Malone, 1976; Nevin \& Shettleworth, 1966).

\footnotetext{
This article is based on a portion of the dissertation submitted by the author to the University of Tennessee at Knoxville graduate school in partial fulfillment of the requirements for a doctoral degree. Requests for reprints should be sent to Timothy $L$. Cleary, Department of Psychology, Illinois College, Jacksonville, IL 62650.
}

Although overall behavioral contrast does not appear to depend on the continuation of a local contrast effect, an important relationship between local contrast and behavioral contrast may still exist. Marcucella and MacDonall (1977) indicated how such a relationship may develop. The researchers looked at the interresponse-time (IRT) distribution of pigeons' pecks in an unchanged component of a multiple random interval (mult RI RI) 60-sec schedule in which one of the components was changed to EXT. The reinforced IRTs became shorter, and the total distribution of IRTs shifted toward shorter intervals. Marcucella and MacDonall suggested, following Mackintosh (1974), that the new IRT distribution (which emphasized short IRTs) was the result of local positive contrast. Once established, the microcontingencies represented by the reinforcement of short IRTs served to maintain the shifted distribution, even though local contrast effects may have no longer been present. The dependence of overall contrast on local contrast would therefore decrease.

To better understand contrast effects, it would be helpful to follow the correlation between the local form and the overall form of behavioral contrast during the course of the development of behavioral contrast. If the precursor mechanism suggested by Marcucella and MacDonall (1977) operates, then the correlation between the two forms should decrease across prolonged discrimination training as overall behavioral contrast becomes independent of a local contrast effect.

However, one of the problems with comparing overall contrast with local contrast is that overall contrast has traditionally been measured against a baseline before discrimination training is begun. The baseline average remains a constant during the discrimination phase and becomes further removed in time and training as discrimination sessions progress. Local contrast, on the other hand, is typically based on session-by-session recordings of response rate. 
To address this problem, the present study used a session-by-session measure of baseline $S+$ response rate to estimate overall positive behavioral contrast. The session baseline measure should be as uninfluenced by the extinction component $(\mathrm{S}-)$ as possible. To accomplish this, pigeons were presented with a three-component multiple schedule in which two of the components were presented with reinforcement and the third was presented in extinction. One of the positive components (component A) was sometimes directly preceded by the extinction component; the other positive component (component B) was not directly preceded by the extinction component. Since component B was never affected by a direct $S-$ to $S+$ transition, it was used as a session-by-session baseline to assess overall rate increases in component $A$ (which was affected by direct $S-$ to $S+$ transitions).

Overall positive behavioral contrast appeared as the differences in response rates between component $A$ and component $B$. Local positive contrast appeared as the differences in response rates between component $A$ when preceded by the extinction component and component $\mathrm{A}$ when preceded by a positive component.

A precursor hypothesis for the origin of behavioral contrast would predict that the appearance of local contrast accompanies the appearance of overall contrast, but as discrimination training continues, overall contrast will become increasingly independent of local contrast. The purpose of the present experiment is to test this prediction.

\section{METHOD}

\section{Subjects}

One Silver King, 1 White Carneau, and 2 Tumbler pigeons, all with varied experimental histories, were maintained at approximately $85 \%$ of their free-feeding weights.

\footnotetext{
Apparatus

The experimental chamber was a $33 \times 26 \mathrm{~cm}$ aluminum and Plexiglas box placed within a $61 \times 61 \times 93 \mathrm{~cm}$ sound-and-light-shielding enclosure. A 1.1-W houselight, centered on the ceiling of the chamber, provided illumination. During 3-sec periods of food magazine operation, a 6-W light illuminated the feeder area. Stimuli were projected on the response key by an in-line digital display unit using a 6.3-V, 1.6-W bulb. A ventilation fan and a white-noise generator masked extraneous noises, and programming and recording equipment were located in a nearby room.

\section{Procedure}

The birds were placed on a three-component multiple schedule. For narrative purposes, the components are termed A, B, and C. Components A and B were presented with a VI 30-sec reinforcement schedule throughout the experiment. To gain a traditional baseline, component $\mathrm{C}$ was presented with a VI 30 -sec reinforcement schedule for the first 11 sessions. For the next 32 sessions, component $C$ was switched to extinction.

The sequence of presentation of each component was random except for three qualifications: (1) Component B never followed component C, (2) component $\mathrm{C}$ never followed component B, and (3) no component was presented more than three times in succession. In the sequence so generated, component A occurred 57 times, component B occurred 38 times, and component $\mathrm{C}$ occurred 33 times. With component durations of $30 \mathrm{sec}$, a session lasted $64 \mathrm{~min}$. Sessions were conducted 6 days a week.
}

\section{RESULTS AND DISCUSSION}

After 11 sessions of baseline, there was little difference in response rates between components. Average response rates in components $A, B$, and $C$ were $29.12,28.44$, and 29.80 , respectively. A one-way repeated measures analysis of variance (ANOVA) failed to find a significant difference in response rates across the components during baseline $[F(2,8)=0.08]$.

On the 12th session, component $C$ was changed from a VI reinforcement schedule to extinction. Response rates in component $\mathbf{C}$ decreased over the succeeding sessions, whereas response rates in component $\mathrm{A}$ and component $\mathrm{B}$ diverged. Figure 1 shows the mean rates of keypecking for component $A$, both when after the extinction component $\mathbf{C}$ and when after a positive component, either $\mathrm{A}$ or $B$. Also shown is the mean rate of keypecking during the positive component $B$. The sample standard deviations are given in parentheses. The mean rates are averages over the 32 sessions of discrimination training.

Responding in A was greater than responding in B, a positive component never immediately preceded by $C$, indicating overall positive behavioral contrast. Additionally, local positive contrast was indicated by responding in component $\mathrm{A}$ being, on the average, greater when it was immediately preceded by component $C$, compared with the condition in which $\mathbf{A}$ was immediately preceded by a nonextinction component. A one-way repeated measures ANOVA across the component types listed in Figure 1 confirmed that the effect of component type was statisti-

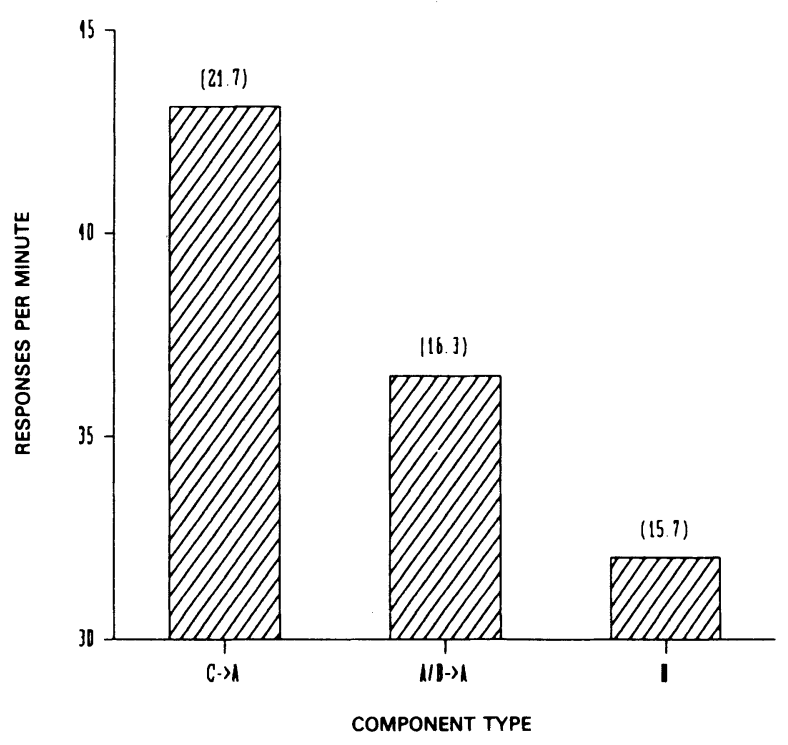

Figure 1. The mean rate of keypecking for component $A$, both when after the extinction component $(C \rightarrow A)$ and when after one of the positive components $(A / B \rightarrow A)$. Also shown is the mean rate of responding in component $\mathrm{B}$, a positive component never immediately preceded by component $\mathrm{C}$. The sample standard deviation for each mean rate is given in parentheses. 


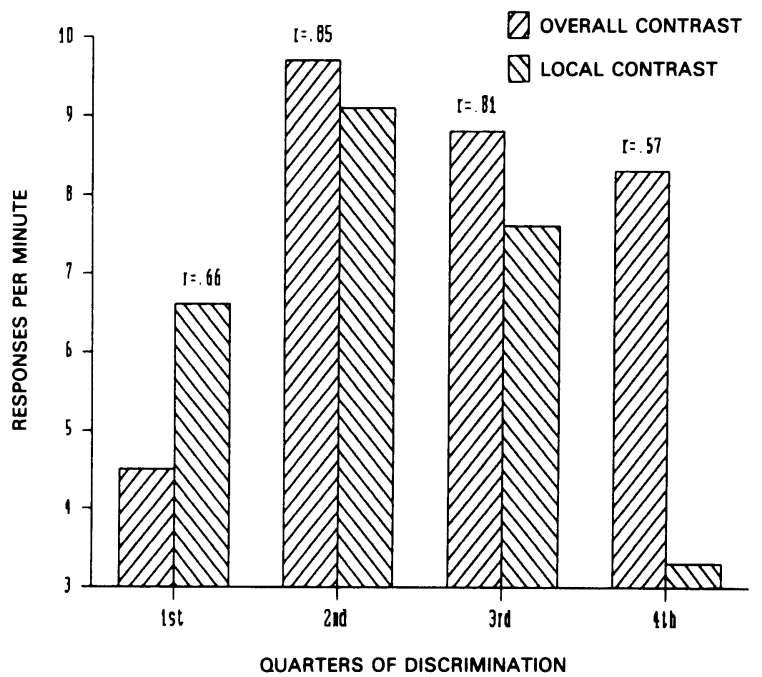

Figure 2. Overall positive behavioral contrast and local positive contrast for each quarter of discrimination training. The correlation between the two measures for each quarter is listed above the respective bars.

cally significant $[F(2,8)=4.94, p<.05]$. A post hoc multiple comparison of group means, using the Bonferroni method suggested by Maxwell (1980), found no significant comparisons.

The correlation between overall behavioral contrast and local contrast was one question to be addressed by this study. Figure 2 shows both the mean overall positive behavioral contrast measure and the mean local positive contrast measure for each quarter of discrimination training (each quarter was eight sessions in duration). The overall behavioral contrast measure is the average response rate in component $\mathrm{A}$ minus the average response rate in component $\mathrm{B}$. The local contrast measure is the response rate in $\mathrm{A}$ after $\mathrm{C}$, minus the response rate in $\mathrm{A}$ when after either B or A. The Pearson product-moment correlation between the measures for all subjects in each quarter is given, and all are significantly different from zero $(p<.05)$.

Local contrast and overall behavioral contrast appeared together in the first quarter of discrimination training, and both effects peaked during the second quarter. The positive correlation between the two measures decreased across the discrimination phase, with the greatest corre- lation seen in the second quarter and the least during the last quarter.

Although local and overall behavioral contrast both appeared early in discrimination training, the positive correlation of the measures was found to weaken across the discrimination phase. The results agree with Marcucella and MacDonall's (1977) precursor analysis of the origin of overall contrast, since the independence of overall contrast from local contrast increased during the discrimination.

One of the difficulties posed by the suggestion that overall behavioral contrast is dependent on local contrast is that overall contrast often remains when local contrast has faded (e.g., Blough, 1988). Precursor theories are attractive because an interacting relationship between local and behavioral contrast may be established without the requirement that local contrast remain in order for overall contrast to continue. A precursor theory may therefore be the most viable view of the relationship between overall and local contrast.

\section{REFERENCES}

BLough, P. M. (1988). Overall and local contrast in multiple schedules: Effects of stimulus similarity and discrimination performance. Animal Learning \& Behavior, 16, 395-403.

Mackintosh, N. J. (1974). The psychology of animal learning. New York: Academic Press.

MACKINTOSH, N. J., LITTLE, L., \& LoRD, J. (1972). Some determinants of behavioral contrast in pigeons and rats. Learning \& Motivation, 3, 148-161.

MaLone, J. C., JR. (1976). Local contrast and Pavlovian induction. Journal of the Experimental Analysis of Behavior, 26, 425-440.

Marcucella, H., \& MacDonall, J. S. (1977). A molecular analysis of multiple schedule interaction: Negative contrast. Journal of the $E x$ perimental Analysis of Behavior, 28, 71-82.

MAXWELl, S. E. (1980). Pairwise multiple comparisons in repeated measures designs. Journal of Educational Statistics, 5, 269-287.

Nevin, J. A., \& ShetTleworth, S. A. (1966). An analysis of contrast effects in multiple schedules. Journal of the Experimental Analysis of Behavior, 9, 305-315.

Pavlov, I. P. (1927). Conditioned reflexes. (G. V. Anrep, Trans.). London: Oxford University Press.

Terrace, H. S. (1966). Stimulus control. In W. K. Honig (Ed.), Operant conditioning: Areas of research and application (pp. 271-344). Englewood Cliffs, NJ: Prentice-Hall.

Williams, B. A. (1983). Another look at contrast in multiple schedules. Journal of the Experimental Analysis of Behavior, 39, 345-384.

(Manuscript received July 17, 1991.) 